\title{
3. CEPHEID VARIABLES IN THE SMALL MAGELLANIC CLOUD
}

\author{
HALTON C. ARP
}

Two-colour light curves have been obtained for sixty cepheids in the SMC. From twenty to thirty-four points have been obtained in each colour. The derived $B$ and $V$ parameters of these variables are estimated to be systematically accurate to $\pm 0^{\mathrm{m}} \mathrm{I}$ with accidental errors of only a few hundredths of a magnitude. These variables all come from a region about $\mathrm{I}^{\circ}$ square in the north-following wing of the SMC, near NGC $37 \mathrm{I}$.

The observed period-apparent magnitude relation for these cepheids is $B_{\text {mean }}=I 7.8$ $-2 \cdot 2 \log P$ (Fig. 5). The zero-point of the cepheid $P-L$ relation may be best discussed in the following way:

I. The apparent distance modulus of the SMC is $m-M=\mathrm{I} 9.2$ from:

(a) Novae, $19 \cdot 2 \pm 0 \cdot 3$

(b) RR Lyrae, I9.2 \pm ? and giant branches of $\mathrm{NGC} 36 \mathrm{I}$ and $4 \mathrm{Ig}$;

(c) main sequence, $19.4 \pm 0.5$.

This yields $B_{\text {mean }}=-\mathrm{r} \cdot 4-2 \cdot 2 \log P$ for SMC cepheids;

$$
B_{\text {mean }}=0 \text { for RR Lyrae cepheids. }
$$

2. The apparent distance modulus of the SMC is $m-M=18.7$ from:

(a) RR Lyrae's at I9.2 $\left(M_{B}=+0 \cdot 5\right)$.

This yields $B_{\text {mean }}=-0 \cdot 9-2 \cdot 2 \log P$ SMC cepheids;

$$
B_{\text {mean }}=+0.5 \quad \text { RR Lyrae cepheids. }
$$

Although the first set of values is the most probable, the second possibility cannot be excluded. Probably the most important thing one could do is to check photo-electrically the magnitude scale which was used to measure the apparent magnitude of the RR Lyrae stars. For example, if the RR Lyrae apparent magnitudes are really fainter, then the intrinsic magnitude of the RR Lyrae stars may be moved fainter while still keeping the I $9 \cdot 2$ modulus.

The observed $P-L$ relation does define a mean line, Fig. 5 , but it also shows a considerable dispersion which is of interest both for purposes of distance determinations and for the theory of cepheid pulsation. In the present observations the total scatter around the mean $P-L$ line is about one magnitude. It is apparent that the distribution of points around the mean $P-L$ line is not symmetrical. Fig. 6 shows a histogram of the projected distribution along the mean $P-L$ line. The dispersion about the line is seen to be not a normal error distribution.

Now it is clear that all extrinsic factors which contribute to the dispersion, with the possible exception of reddening, are negligible. For example, a volume with an apparent diameter of $2^{\circ}$ gives a back to front magnitude difference of only $0^{\mathrm{m}} \mathrm{O} 8$. Cepheids which have unresolved companions are readily recognizable by the effect on the shape of the light-curve.

There are two remaining possible causes for the observed scatter in the $P-L$ relation, intrinsic dispersion and reddening.

Intrinsic dispersion in the luminosity of cepheids for a given period: using only the general relation for oscillating systems $P \rho^{\mathrm{m}}=$ constant, the mass-luminosity law, and the relation between radius, temperature and colour index, and empirical $P-L$ relation may be computed [r]. Because of the finite width of the region of cepheid instability in the H-R diagram, a cepheid passing through the region at a constant luminosity may have differing colour indices and therefore different periods at a given luminosity. Sandage has computed the expected $P-L$ relation using the relation between $V$ and $B-V$ for the present cepheids. The slope of the $P-L$ relation found here is reproduced well and, in particular, a dispersion of about one magnitude is derived. If more than one mode of oscillation is permitted, then the total dispersion is slightly larger, but the dispersions around each modal line are smaller. In either case, the arguments in favour of interpreting the distribution about the $P-L$ line here as intrinsic dispersion are:

I. A peculiar distribution of reddening is needed to account for the bi-modal distribution of magnitude residuals observed in Fig. 6 . It is rather more probable that the 


\section{LUMINOSITY OF CEPHEIDS}

second group of bright stars (about Io \% by number) are vibrating in their first over-tone. Their separation from the main $P-L$ line is slightly less than a factor of 2 , near the expected over-tone value.

2. Most of the extreme deviations from the $P-L$ line have light-curves which are clearly different, in the sense of being more symmetrical.

The computations predict that in the case of intrinsic dispersion the bluer colours belong to the brighter cepheids. This is the same sense that would obtain if the cepheids were dispersed from a discrete $P-L$ line by variable reddening. The data here show a strong

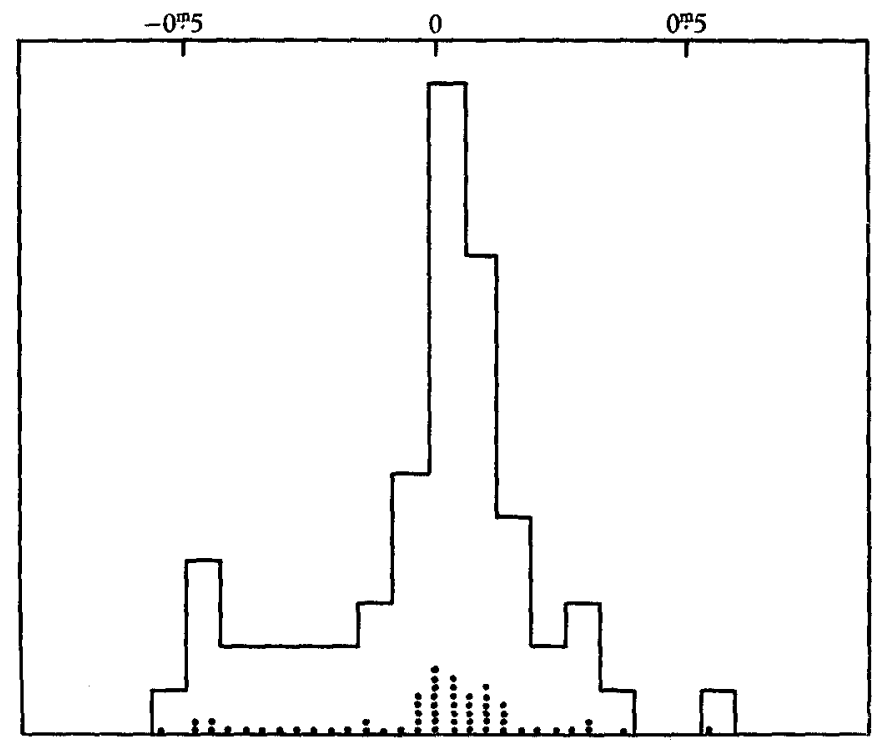

Fig. 6. Distribution of deviations from mean $P-L$ line of Fig. 5 (Arp stars only).

correlation between $\Delta(B-V)$ and $\Delta$ magnitude. Sandage computed that the ratio of reddening to magnitude deviation was very close to the usual ratio of reddening to absorption [r]. Therefore it is not yet possible, in the preliminary analysis of this data, to use the present correlation between deviation in colour and magnitude to distinguish between these two effects.

As for reddening in the field from other considerations, three-colour measures of several super-giants in the region indicate that they are unreddened. The main sequence in this region has a fairly small spread and therefore would not support differential reddening of more than a few tenths of a magnitude.

To consider a second major question, we may ask if there are any certain differences observed between these SMC cepheids and cepheids in our own Galaxy. Aside from the previously known difference in frequency distribution of periods (maximum SMC 2 days, maximum galaxy $4-5$ days), there are now two more differences to be added:

x. The period-amplitude relations (Fig. 4) of the SMC and galactic cepheids agree well from long periods to 7 or 8 day periods. At shorter periods the cepheids in our Galaxy (and in recently investigated galactic clusters) continue to diminish in amplitude. The cepheids in the SMC, however, increase their amplitude, and by the 2 -day periods the SMC cepheids have even larger amplitudes than cepheids of this period in globular clusters. 


\section{JOINT DISCUSSION}

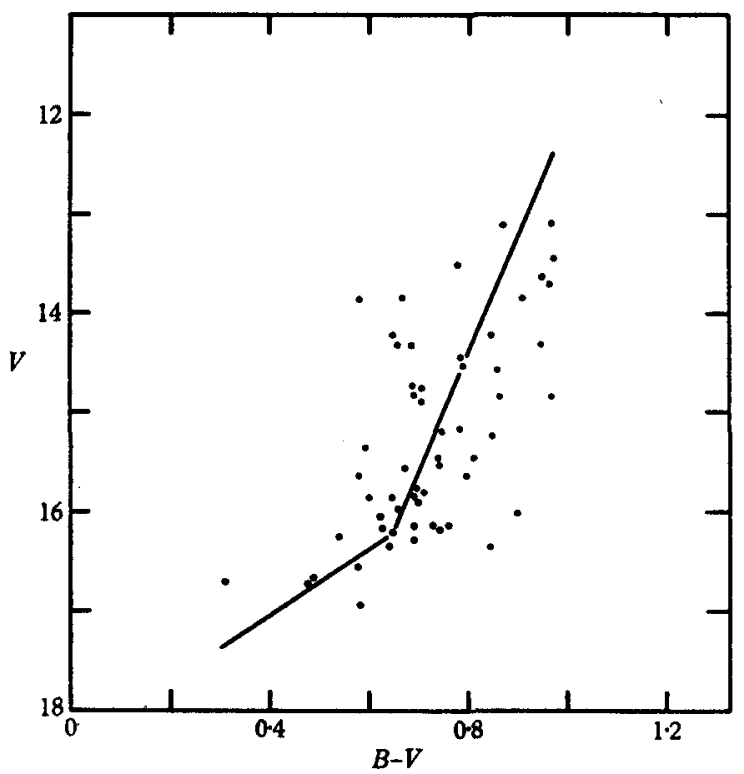

Fig. 7. Place of SMC cepheids in colour-magnitude diagram at mean light (Arp stars only).

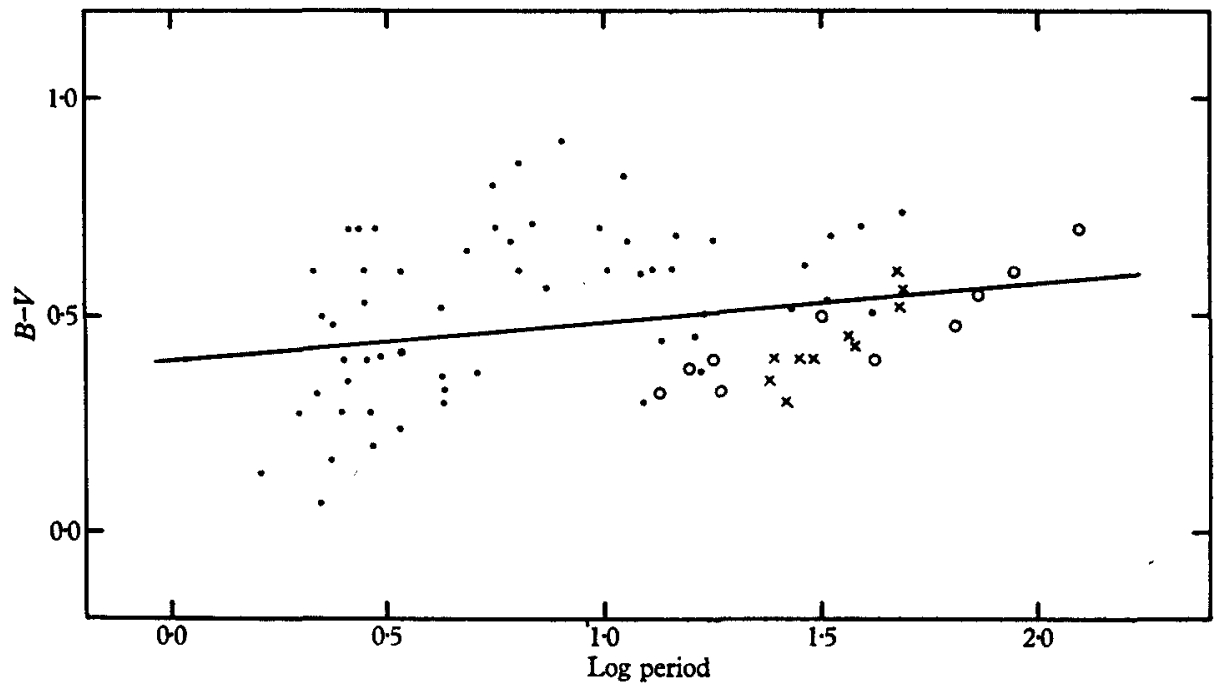

Fig. 8. Period-colour relation for Magellanic Cloud cepheids. Colour index at maximum light. Coding as in Fig. 4. Gascoigne's SMC cepheids come, with one exception, from the leading edge of the Cloud. Whether the slightly bluer colour for these latter variables is due to different average reddening is not yet certain. The line refers to cepheids in galactic clusters and is taken from Fig. I (b). 


\title{
LUMINOSITY OF CEPHEIDS
}

2. The colour-magnitude relation follows the period-amplitude relation and increases again after the 7-8 day period for the SMC cepheids, in contrast to the behaviour in the Galaxy (Fig. 7).

The mean colour indices for SMC cepheids, galactic cepheids in clusters (less than 8 days period) and cepheids in globular clusters all agree to within $\pm 0^{m} \mathrm{I}$, however, in the present measures (Fig. 8).

\section{REFERE NCE}

[I] Sandage, A. R. Ap. J. 127, 513, r958.

\section{THE INTRINSIC COLOURS OF CEPHEID VARIABLES}

\author{
GERALD E. KRON
}

In I95I, S. C. B. Gascoigne and I, working at the Mount Stromlo Observatory in Australia, found that variable stars [r] in the Small Magellanic Cloud were apparently much bluer than classical cepheids of equivalent period in the Galaxy. It therefore became important to know if this colour difference was a property of the stars, or if it was caused by different space reddening.

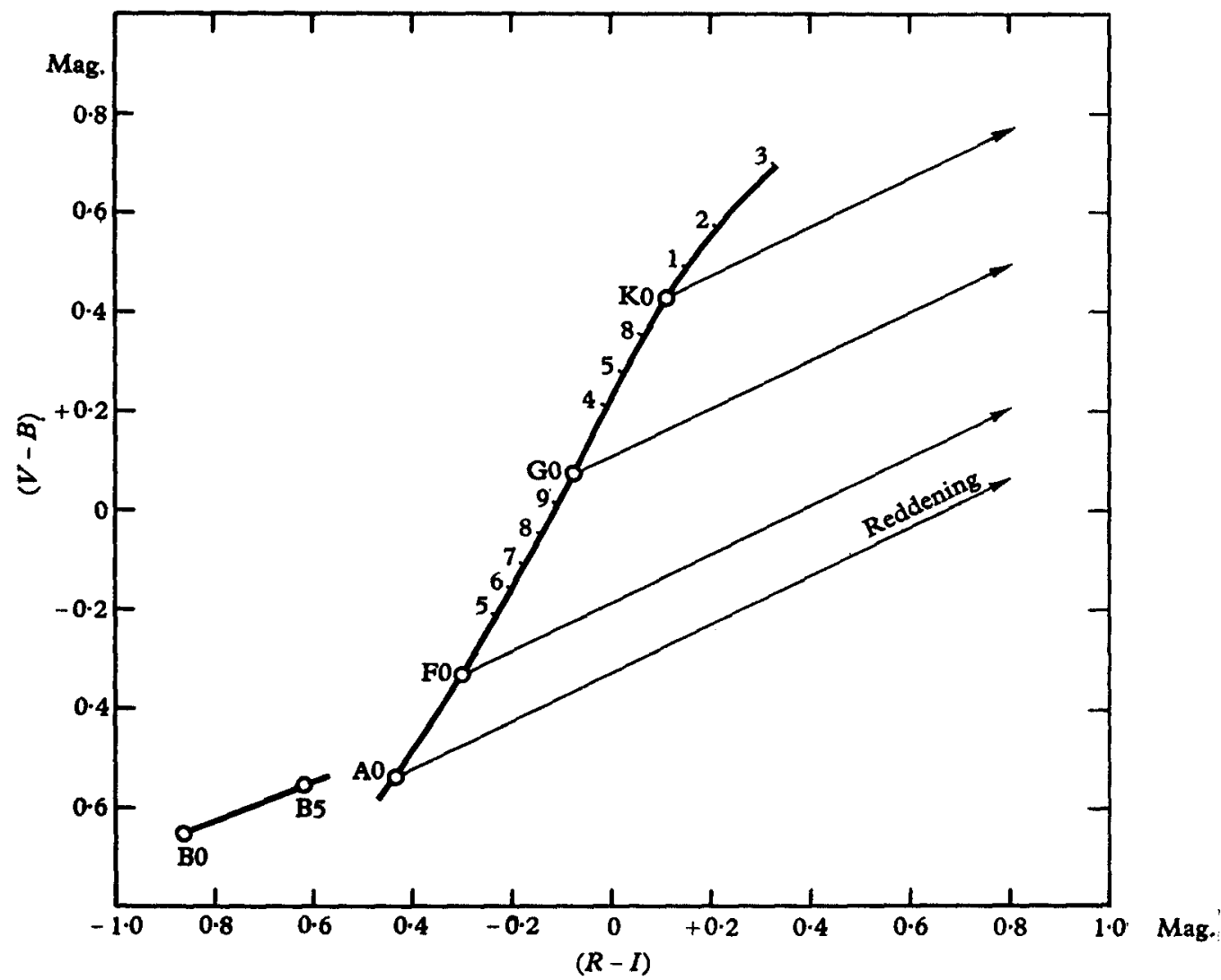

Fig. 9. Calibration curve for determining the reddening of cepheid variables by means of six-colour photometry. Two-colour differences are formed from four of the six colours, the other two not being used. 